\title{
The Design of a Configurable, Extensible and Dynamic Notification Service
}

\author{
Roberto S. Silva Filho ${ }^{1} \quad$ Cleidson R. B. de Souza ${ }^{1,2} \quad$ David F. Redmiles ${ }^{1}$ \\ ${ }^{1}$ School of Information and Computer Science \\ University of California, Irvine \\ Irvine, CA, USA \\ ${ }^{2}$ Informatics Department \\ +1949 824-4121 \\ Federal University of Pará \\ Belém, PA, Brazil \\ +55 91 211-1405 \\ \{rsilvafi, cdesouza, redmiles\}@ics.uci.edu
}

\begin{abstract}
Publish/subscribe infrastructures, specifically notification servers, are used in a large spectrum of distributed applications as their basic communication and integration infrastructure. With their recent popularization, notification servers are being developed to support specific application domains. At the same time, generalpurpose notification servers provide a large set of functionality for a broad set of applications. With so many options, developers face the dilemma of choosing between application-specific or generalpurpose notification servers. In both cases, however, the set of features provided by the servers are usually neither extensible nor configurable, making their customization to specific application domains a difficult task. In this work, a more flexible approach is proposed - a customizable, extensible and dynamic architecture for notification services - which allows the customization of the notification service to different application domains. The extensibility model is presented according to the design framework proposed by Rosemblum and Wolf. A preliminary implementation of the prototype is also discussed, as well as configuration examples.
\end{abstract}

\section{Categories and Subject Descriptors}

D.2.11 [Software Architectures]: Domain Specific Architectures; D.2.12 [Interoperability]: Distributed Objects; H.4.3 [Communications Applications]: Information Browsers;

\section{General Terms \\ Design}

\section{Keywords}

Notification servers, event-based middleware, dynamic architecture, pluggable architecture.

\section{INTRODUCTION}

Over the last few years, a large number of publish/subscribe services, especially in the form of notification servers, have been used as the basic infrastructure for the implementation of many distributed applications such as user and software monitoring [1], groupware applications [2], awareness tools [3], workflow management systems and mobile applications [4]. Hence, due to its increased popularity, event notification services need to cope with new requirements, coming from different application domains. In fact, a broad spectrum of research and commercial tools are available nowadays. At one extreme, "one-size-fits-all" approaches, such as adopted by CORBA Notification Service (CORBA-NS) [5] or READY [6] strives to address new applications requirements by providing a very comprehensive set of features, able to support a broad set of applications. At the other extreme, specialized notification servers tailored to application-specific requirements provide novel but specific functionalities. Examples of such specialized systems include Khronica [7] and CASSIUS [8] which are specially designed to support groupware and awareness applications; or even Yeast [9] and GEM [10] which are specialized in advanced event processing for local networks applications, and distributed applications monitoring respectively. Finally, servers such as Siena [11] and Elvin [12], even though designed with special domains in mind, strive for a balance between specificity and expressiveness of the subscription language they support.

Therefore, in the development of distributed applications, developers face the dilemma of specialization versus generalization: to use a generalized infrastructure, that can support and integrate different applications, but may not provide all the necessary functionality for specific application domains; or to use one eventbased infrastructure for each application domain, having "the right tool for the right problem", but loosing the uniformity and integration of a single solution. For example, in the development of awareness tools, one can use either a comprehensive solution such as CORBA Notification Service (CORBA-NS) that provides a large set of features and services, or a domain specific solution such as CASSIUS, that provides services for awareness applications. That's important to note that infrastructures as CORBA-NS, even though are very comprehensive in its set of features, do not provide direct support for all the requirements of awareness applications. It does not allow, for example, the browsing and discovery of the event source hierarchy. CASSIUS, on the other hand, provides this feature and allows the easy discovery of information sources and the subscription for events from different source components. If CORBA-NS were used in an awareness application demanding this feature, this service would need to be implemented in the application itself. 
Another problem of the currently available event-based infrastructures is the poor support for selection and customization of the services to be provided, which is important for applications that run on resource-limited devices such as the ones common to mobile applications. If a notification service as CORBA-NS is used for such applications, the whole set of features provided by this server would have to be available for use, whether the application requires it or not. Moreover, current event-based infrastructures lack mechanisms to support the extension of their functionality. The only extension mechanism is usually the (understanding and) change of their source code, or the implementation of the service by the application itself. Additionally, due to restrictions in their event or subscription models, the addition of new functionality may constitute a very difficult task [13].

In this work, we describe the design and implementation of an extensible and pluggable architecture for notification services that provides an alternative to the generalized versus specialized dilemma. It provides extensible event, notification, subscription and resource models (see [14] for a detailed description of these models), which at the same time supports the specialization, extension, and customization of the event notification service toward the needs of different applications. In order to provide this flexibility, we use the following main design strategies: a publish/subscribe core; the combination of this core with plug-ins; the ability to add consumer and producer side plug-ins and services, with the ability to customize their location; the adoption of extensible subscription and event languages. These strategies provide a basic architecture that can be customized and extended to support functionality demanded by current or new application domains. It is also dynamic, allowing the addition and removal of services at runtime.

The paper is organized in the following way: the next section presents, in more detail, the diverse requirements of different application domains; section 3 discusses the analytical design framework used to understand the extensibility requirements of event notification servers; in section 4 we present the design of our architecture; in section 5, we discuss the extensibility of the system; in section 6 we demonstrate the extensibility and configurability of the architecture, presenting two examples; In section 7 we discuss some related work; and finally, in section 8 we present some conclusions and future work.

\section{APPLICATION DOMAINS}

In order to understand the extensibility requirement, it is important to understand the diverse set of requirements from each application domain. In this section, we motivate this aspect, presenting the requirements for three application domains as follows: application and usability monitoring, awareness applications and mobility. Different application domains require specific features from the publish/subscribe infrastructure. We classify these features in functional and non-functional requirements.

For example, event-based application and usability monitoring infrastructures such as EDEM [1] and EBBA [15], provide a common set of features such as event sequence detection; event abstraction - ability to compose new events based on a pattern of events; content-based filtering - ability to define subscriptions based on the event attributes and types; browsing of information sources and their events; and event persistency. All these features are functional requirements.
Awareness applications usually require event persistency and typing of events, event validity (time-to-live), event sequence detection, and different notification delivery mechanisms. Moreover, a special feature in this case is the possibility to browse, and later subscribe to the event types that are published by each event source. This service, called event browsing, provides information about the publishers and their event types currently supported by the server. Example of systems that provide this services are Khronika [7] and CASSIUS [8].

Mobility is another domain with specific requirements. In order to be able to support mobile applications, a notification server needs to provide: simultaneous push and pull interaction policies (for publishers and subscribers); and event persistency (to cope with pull policies and disconnection), all functional requirements. Nonfunctional requirements such as roaming (ability to change the local address due to migration) and security (cryptography and authentication) are also required. Mobility itself can be considered a non-functional requirement for comprising a set of policies and functionalities that together provide this characteristic to the system. JEDI is an example of notification server that provides some of these features [4].

\section{ANALYTICAL DESIGN FRAMEWORK}

In order to approach the design of notification servers, Rosenblum and Wolf [14] propose a framework that captures the most relevant design dimensions (or models) of those systems. In this framework, the object model describes the components that receive notifications (subscribers) and generate events (publishers); The event model describes the representation and characteristics of the events; the notification model is concerned with the way the events are delivered to the subscribers; the observation model describes the mechanisms used to express interest in occurrences of events; the timing model is concerned with the casual and temporal relations between the events; the resource model defines where in the distributed system architecture, the observation and notification computations are located, as well as how they are allocated and accounted; finally, the naming model is concerned with the location of objects, events and subscriptions in the model.

In our design, we will consider a specialization of this framework which is similar to the one adopted by Cugola et al. at [4]. In special, in our model, the naming and observation models are combined together in what we describe as subscription model. In this case, the way resources are identified (naming) is part of the subscription language. A protocol model is defined in order to describe other kinds of interaction with the service other than the basic publish and subscribe messages, allowing the server to support new interaction protocols.

\section{DESIGN}

In order to support the whole spectrum of functional and nonfunctional requirements listed in section 2 , with the flexibility to select the subset of features needed by each application domain, our event notification architecture must be configurable and extensible. This section describes our approach to provide such flexibility. One of the main challenges in our design was to specify an infrastructure that allows the addition of new event processing functions (functional requirements) such as event filtering, sequence detection and abstraction, while allowing the incorpora- 
tion of generalized (non-functional) aspects such as support for security and mobility.

The extensibility needs to embrace all the design dimensions presented in the design framework. The basic elements used in such extensibility are the representation of events, subscriptions and messages in an extensible language (XML); the use of smart parsers and the dynamic instantiation of plug-ins to attend the requirements of each subscription. This section will describe in more detail the extension mechanisms for each one of those models.

\subsection{Architecture overview}

The high-level architecture of the system is presented in Figure 1. In this architecture, a publish/subscribe core allows the extension of the notification, event and subscription models. One of the key elements to this extension is the use of plug-ins. The subscription query and notification mechanism are performed using a dynamic combination of plug-ins with a simple publish/subscribe core.

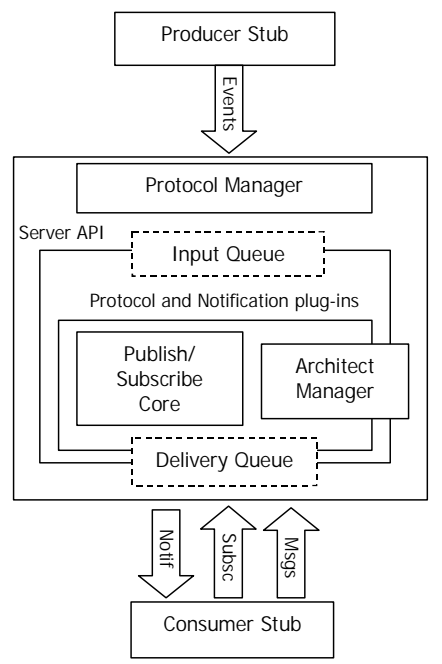

Figure 1: The high-level architecture of the extensible and configurable notification server.

The architect manager orchestrates the addition and removal of plug-ins, and the allocation of components throughout the system, providing runtime change capabilities to the resource model. The protocol manager provides an extension point to handle not only the publish/subscribe messages, but also every other communication as, for example, those related to the security (authentication) and mobility (roaming) protocols. Each protocol is provided by a specific plug-in added to this component.

Non-functional requirements are addressed by the combination of those mechanisms in configurations that are instantiated by the architect manager. Each one of those approaches and main component of the architecture is described in more detail as follows.

\subsection{The publish/subscribe core}

The publish/subscribe core, described in Figure 2, is defined by a set of sub-components that make possible the handling of different subscriptions, using the available plug-ins.

Subscription Parser. Subscriptions are defined as messages according to an extensible XML schema (or grammar), which allows the definition of queries and event notification options. The subscription parser validates and extracts the subscription queries and delivery options from subscription messages, forwarding this information to the subscription and notification managers respectively as depicted by arrows (1), (2) and (3) in Figure 2.

Subscription Manager. The subscription manager component is responsible for handling the subscription queries provided by information consumers. It performs the interpretation of these requests assembling subscription trees that will execute the appropriate query using different plug-in instances, created with the help of the plug-in manager component (4) and (5).

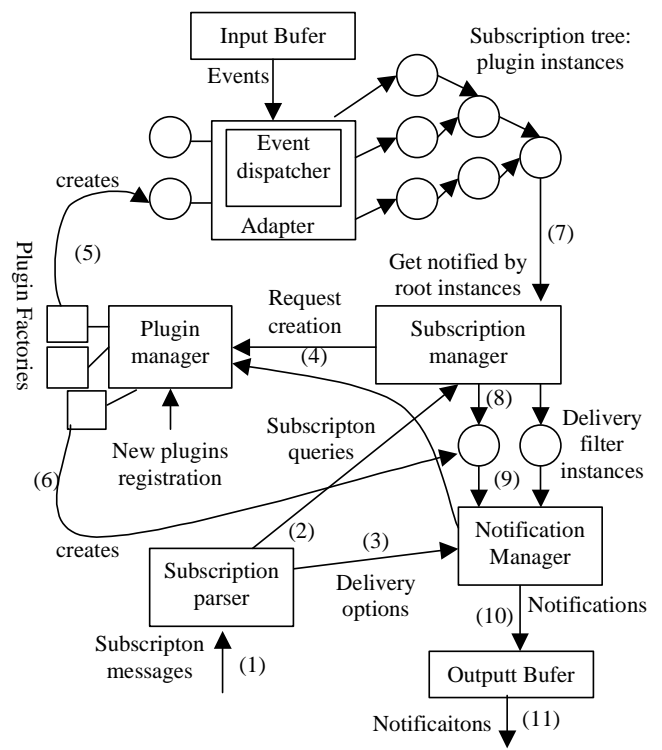

Figure 2 Architecture of the publish/subscribe core.

For example, Figure 3 describes the subscription tree produced by the parsing of the following expression (note that, in this example, an algebraic form is used instead of XML for clarity and readability):
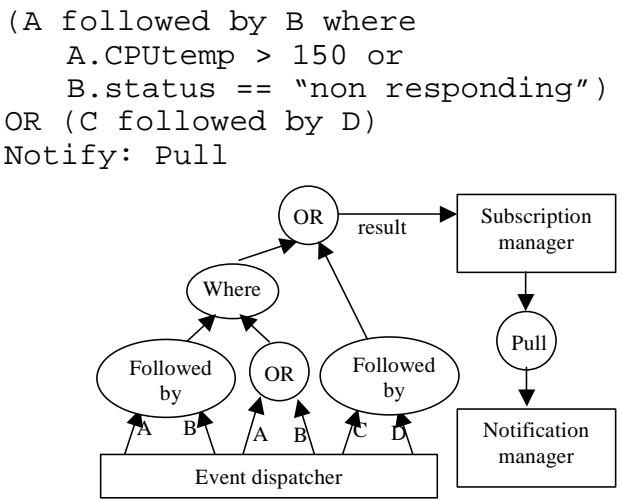

Figure 3 Decomposition of subscriptions by the subscription and notification managers using plug-ins.

As depicted in Figure 3, the subscription evaluation tree is composed of nodes, which are plug-in instances that communicate using the simple publish/subscribe design pattern [16]. In this tree, each level subscribes to its children nodes' results. A subscription like "A.CPUtemp > 150 or B.status == 'non responding'", for example, is evaluated by an OR 
plug-in instance that subscribes to events of type A and B. Once the tree is assembled and configured, the subscription manager subscribes itself to the results of each tree root plug-in (7). These results can be Boolean events, showing the occurrence or not of the subscription, a new event produced due to the evaluation of an expression, or a set of events that matched the specific filter.

This approach also allows the subscription manager to perform optimizations such as the reuse of sub-expression parts between two or more subscriptions. For example, in an expression such as: "(A followed by B or C)" and another expression "( followed by B or C)", the 'OR' part of is common to both subscriptions. Since the evaluation uses the publish/subscribe model as described in Figure 3, subscription trees can be easily rearranged to share the same 'OR plug-in' output event.

Notification Manager. Similarly to the subscription manager, the notification manager is responsible for parsing the notification options and allocating the appropriate plug-ins for the delivery of notification (4) and (6). For example, in Figure 3, pull notification is selected, which will be handled by the appropriate pull delivery plug-in.

Plug-in Manager. In order to assembly subscription and notification evaluation trees, both the subscription and notification managers use the plug-ins registered in the plug-in manager. Each plug-in is created by a registered factory [16], which is indexed by one or more operators (XML tags) that the plug-in is able to evaluate. For example, a '<FOLLOWED BY>' plug-in processes the ordered event sequences, being indexed by that special XML tag.

Event Dispatcher. A simple event dispatcher provides the basic event routing mechanism and dictates the event model of the notification server. If a content-based dispatcher is provided, and the event model is tuple-based, all the components will share this basic functionality and the event language will be built on this basic foundation. In case an off-the-shelf event dispatcher is used, an adapter provides a standardized and simple publish/subscribe API that hides the idiosyncrasies of the selected component, including its event and subscription models.

\subsection{Protocol Manager}

Non-functional services such as security and mobility usually demand other forms of interaction with the server, other than the basic publish or subscribe commands. For example, security usually requires an authentication protocol whereas mobility may require the extension of the server protocol with move-in/moveout commands [4]. The ability to support these and other additional services requires a mechanism to provide new interaction mechanisms to the server. The protocol manager works as a message router, allowing the registration of plug-ins to handle each protocol. This component deals with aspects related to the protocol model defined in section 3 .

\subsection{Architect manager}

The architect manager is the component responsible for the configuration of the diverse services and plug-ins in the architecture. It reads an XML specification with the topological arrangement of the components, and the list of available plug-ins, performing their proper installation in the system.
Runtime changes in the plug-in factories and the components of the system are also managed by this component. The addition of new plug-ins consists on the registration of their factories in the plug-in manager. This process may require the interruption of the event flow. For such the input and delivery queues can be temporarily paused, while the new service is inserted in its appropriate place.

\section{EXTENSIBILITY}

In order to be extensible, the architecture needs to support variations and extensions to the different models described in section 3 . This is usually provided by a combination of language extensions and plug-ins and configuration of components. This section discusses the strategies used to extend each one of the models.

\subsection{The Event model}

Events can be represented in many different ways. The most popular forms are tuples, records and objects [4]. The event model used by the system impacts on the possible extensions the system will be able to have. For example, type checking is a feature available in record or object-based models, but not in tuple-based systems. Hence, the choice of one of these models is dependent on the requirements the system will be customized to attend.

The event model is a native property of the event dispatcher and should be matched by a subscription language and a special plugin to handle it. For example, if Siena [11] is used as the event dispatcher, the event model of the system will be tuple-based. The Siena subscription, which is content-based, will need to be described according to an XML schema, as well as the Siena event. A special plug-in, registered with the plug-in manager to handle the tags in this language, matching it with the provided event, will also have to be provided.

In order to extend or customize the event model, the event language and the event dispatcher adapter have to be customized. For example, a tuple-based publish/subscribe core can provide types. In this case, types are special attributes in the tuple-based event, and should be enforced (type checking) and managed (type declaration) by the adapter.

\subsection{The subscription model}

In order to be able to handle different functionalities, the subscription language of the server is extensible. It is described according to an XML schema that defines a set of basic tags that can be extended to provide new functionality. For example, support for out of order event detection can be expressed by the addition of a new element in the language: "( $A$ or-sequence $B)$ " which is mapped to a special tag which use is illustrated as follows:

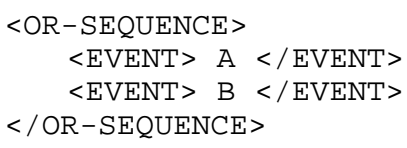

The occurrence of this new tag in a subscription, will instruct the subscription manager to look-up the '<OR-SEQUENCE>' tag in the plug-in manager. The correspondent plug-in for this tag is instantiated and the whole expression between <ORSEQUENCE> and </OR-SEQUENCE> is passed to this plug-in, which implements the subscription command. Plug-ins may need results from other plug-ins, which issues in the subscription evaluation tree in Figure 3. 
In case a tag is not registered, indicating that its corresponding plug-in factory is not installed, the plug-in manager may redirect the request to a generic plug-in that can search the plug-in implementation repository, or the Web, for that specific factory implementation. If available, the service is downloaded and installed at runtime, allowing the plug-in instance to successfully build an expression evaluation tree. In case of failure, the subscription process is not completed, and an error control event is produced.

An example of an event processing language and service, with a configuration focusing on the support for awareness application, is discussed in [17].

\subsection{The notification model}

Extensions to this model are defined in the same way as the subscription model. For each new notification language extension, an XML schema is provided, together with a plug-in to interpret that extension tags. Once the plug-in is installed, the notification manager can now use it in a publish/subscribe evaluation tree. Example of notification plug-ins includes push or pull delivery and event persistency.

\subsection{The protocol model}

A protocol language is described as a set of primitive messages defined in XML. For each protocol, a plug-in is defined to orchestrate and handle the use of this set of messages. In an authentication protocol extension, for example, an authenticator plug-in will be responsible for the validation of the system users, the management of the authorized users database, and the denial or concession of access to each user to the set of events and subscriptions of the system.

\subsection{The resource model}

Some application domains may require the execution of part of the subscription activities in the producer or consumer sides. This approach usually results in the distribution of processing and the reduction of messages exchanges with the central service, which is important in mobile, software monitoring or general large-scale applications, for example. Our model copes with these requirements by permitting the optional partial execution of subscriptions in both the producer and consumer processes.

Consumer and producer stubs mediate the interaction of the application with the notification service. Publishers interact with their stubs in order to send events, whereas consumers provide subscriptions and implement a listener interface defined in the consumer stubs.

\subsubsection{Publisher and Consumer plug-ins and filters}

Figure 4 shows an example of a subscriber stub internal architecture that can be extended using plug-ins and notification filters. The use of a local subscription manager component allows the evaluation of subscriptions in the consumer address space. The purpose of this evaluation at the client-side permits, for example, that only well-formed subscriptions get to the server. This is helped by local instances of the plug-in manager and the subscription manager components.

Note that similar to the event dispatcher core, this consumer stub uses the simpler listener design pattern to assembly subscription evaluation trees locally. Simple events are received from the notification server. Functions as AND's, OR's or sequence detec- tion, for example, are executed locally based on these events. Again, this is an optional feature that our architecture supports: the distribution of the event processing in the consumers or publishers. Of course, a developer might choose not use this feature and let the server process the subscriptions.

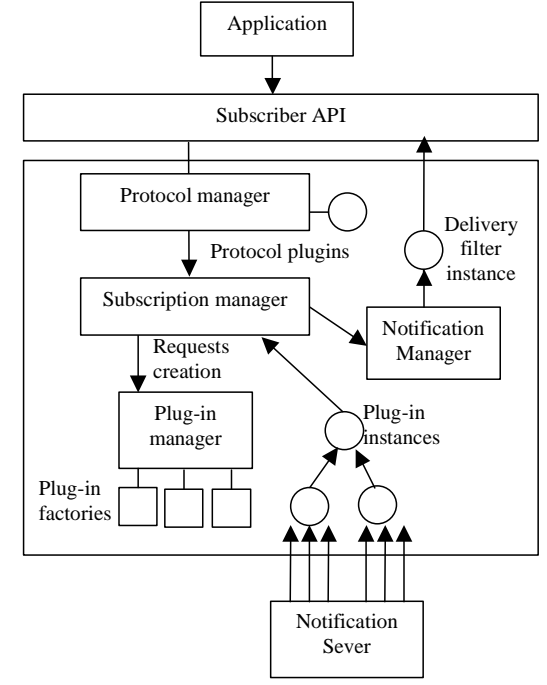

Figure 4 An example of the pluggable consumer stub configuration.

Protocol plug-ins can be defined in order to implement client side security and mobility services. Another option is the local use of notification plug-ins. For example, producer-side persistency is useful in some mobile applications where events can be posted for delivery during disconnected operation, being sent when the connection to the notification server is reestablished.

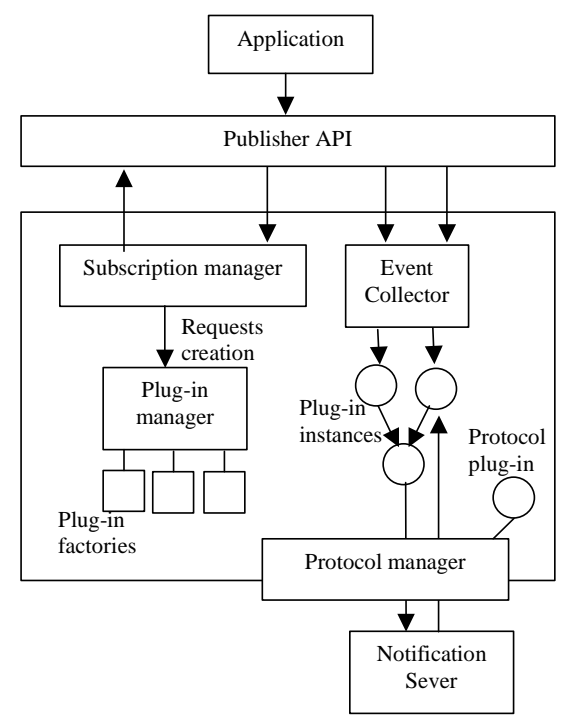

Figure 5 An example of a publisher stub configuration.

In another example, described in Figure 5, a publisher stub is extended with plug-ins used to collect event sequences and send higher-level events when the condition being evaluated is satisfied. In other words, these are composition or abstraction plug-ins. This approach requires download and execution of part of the subscription locally, those parts of the subscription evaluation tree that uses events produced in the current process. This does not 
limit the scope of the subscriptions, since events coming from other applications can be combined with local events, during expression evaluations, by allowing plug-in instances to subscribe to these events in the notification server. For example, for performance reasons, one can decide to do the evaluation of the following subscription in the publisher side: " $\mathrm{A}$ and $\mathrm{B}$ and $\mathrm{C}$ and $\mathrm{D}$ and E)", where A through D are locally produced events and E is produced by another application elsewhere.

The use of publisher plug-ins makes sense only when most of the events being processed come from the local host, as it is the case of event monitoring performed by systems like EDEM.

\subsection{Extensibility summary}

Table 1 presents the main design dimensions addressed in the system design, with their extension mechanism. Examples of features that can be added to the system using this approach are also presented.

Table 1 Adaptation points and features to extend according to the notification service model

\begin{tabular}{|l|l|l|}
\hline \multicolumn{1}{|c|}{ Design dimension } & \multicolumn{1}{|c|}{ How to Extend } & \multicolumn{1}{|c|}{ Examples } \\
\hline $\begin{array}{l}\text { Subscription (or } \\
\text { event query) model }\end{array}$ & $\begin{array}{l}\text { Extensible subscription language } \\
\text { Provide feature specific event processing plug-ins }\end{array}$ & $\begin{array}{l}\text { Event aggregation } \\
\text { Abstraction } \\
\text { Sequence detection }\end{array}$ \\
\hline Event Model & $\begin{array}{l}\text { Extensible event representation language } \\
\text { Provide an event adapter for each dispatcher used } \\
\text { Provide a plug-in to handle the dispatcher specific event language }\end{array}$ & $\begin{array}{l}\text { Tuple based } \\
\text { Record based (with event typing) } \\
\text { Object based (with event typing) }\end{array}$ \\
\hline Notification Model & $\begin{array}{l}\text { Notification plug-ins (or filters) } \\
\text { Extensible subscription language that allows the definition of notification } \\
\text { policies }\end{array}$ & $\begin{array}{l}\text { Push } \\
\text { Pull (with persistency) }\end{array}$ \\
\hline Resource Model & $\begin{array}{l}\text { Server configuration language and configuration manager that allows the } \\
\text { distribution of event processing to server-side or client-side plug-ins }\end{array}$ & $\begin{array}{l}\text { Centralized } \\
\text { Partially distributed }\end{array}$ \\
\hline Protocol Model & $\begin{array}{l}\text { Extensible protocol language } \\
\text { Protocol plug-ins to handle different protocols }\end{array}$ & $\begin{array}{l}\text { Security protocols } \\
\text { Mobility protocols } \\
\text { Configuration protocols }\end{array}$ \\
\hline
\end{tabular}

\subsection{Implementation}

A prototype of the event-notification server architecture described in this work is being implemented in Java (Sun J2SDK1.4). The server side components, including the plug-in infrastructure, were implemented, as well as a simple version of the architect manager.

In our prototype, many simple content-based publish/subscribe systems could be used as the event dispatcher component in the publish/subscribe core. We are currently using Siena as this component for its simple tuple-based event model, and its ability to match content-based subscriptions.

Extensions to the basic Siena functionality as sequence detection and event rules were implemented using this framework. Other plug-ins are being developed to provide the functionality of CASSIUS [8] and the EDEM [1] event language in our architecture.

The extension of the core functionality with simple plug-ins is relatively easy. It requires the implementation of a component according to the plug-in interface and the extension of the subscription language to include the expressions (with their tags) that express the new functionality. The plug-in location is then informed to the system through the architect manager. Of course, more advanced features such as "rule" extension plug-ins, will require more complex code, than a simple sequence detector.

In parallel to the design and implementation of the system, we are also focusing in the subscription language to be used in our eventnotification service. A language to address the specific problems of CSCW applications was proposed in [17]. A low-level representation of this language, using XML, is being developed.

\section{EXAMPLE CONFIGURATIONS}

In this section we present two examples of configurations of our architecture supporting representative application domains that use event-notification services, namely application and usability monitoring and awareness. These configurations were created to support features provided by notification servers used in these domains. A configuration of the architecture is a particular disposition of its components in order to attain a specific set of requirements or features. By presenting these configurations, we show that the elements of our architecture can be easily customized to implement application-specific notification servers.

\subsection{Application and usability monitoring}

In this application domain, software components and GUI design are monitored in order to detect design flaws and misassumptions with actual prototypes of the system being analyzed. In this context, an event-notification server needs to support the following subset of features: event sequence detection; event abstraction; content-based filtering; browsing of information sources and their events; and event persistency as a support for pull delivery of notifications. These features are provided by event monitoring applications as EDEM [1] and EBBA [15].

In this example, the publish/subscribe core of our architecture already provides content-based filtering. Sequence detection and event abstraction are implemented as plug-ins used by the subscription manager to extend the subscription model. In the EDEM approach, as discussed in section 5.5.1, the plug-ins are installed in the publisher stub; whereas in the EBBA approach, they are 
installed in the consumer stub. Our architecture supports both cases, since it allows plug-ins in both producer and consumer stubs. Furthermore, it supports the dynamic change from one approach to the other.

Event types are assured and implemented as an extension to the tuple-based model of the core. This is implemented in the event dispatcher adapter, and as an extension to the dispatcher language, provided by the event dispatcher plug-in. Persistency and pull delivery are plug-ins used by the notification manager. The browsing of information sources and their events is performed by plug-ins used by the protocol manager.

\subsection{Awareness applications}

Notification servers such as Khronika [7] and CASSIUS [8] are specially designed to support the development of awareness applications. These servers provide event persistency and typing, event validity (time-to-live), event sequence detection, and different notification delivery mechanisms. Moreover, a special feature provided by CASSIUS is the ability to browse, and later subscribe to the event types that are published by each event source. This service, called event browsing provides information about the publishers and their event types currently supported by the server.

Event typing is provided by the event dispatcher adapter, which may extend the event model of the publish/subscribe core to provide this facility. Type checking is also provided by this component. The other features are provided by a set of plug-ins. Event browsing comprises the ability to advertise event sources types (like in Siena), organize this information in a database and provide this information to event consumers. This functionality is provided by protocol plug-ins. On the publisher side, another event browsing protocol plug-in is installed, forwarding queries about the event hierarchy to the server. Similarly to mobile applications, delivery policies and persistency are implemented as notification plug-ins. Content-based filtering is already addressed in the publish/subscribe core, but sequence detection is provided as a subscription query plug-in.

\section{RELATED WORK}

The general idea of extensible and configurable software architectures is not new, being a research topic in different computer science fields. This section explores some of the previous work that inspired our approach.

\subsection{Pluggable and programmable routers}

The Click Modular Router [18] defines a basic architecture for the definition of flexible and modular Internet routers. In this architecture, software modules can be arranged according to an IP routing workflow, allowing the expression of different policies and configurations that coordinate the proper routing of IP packages. Promile [19] is another system that extends the Click Modular Router configurable architecture, adding to it the runtime change capability. It uses a graph (workflow), described in XML, to specify the interconnection between modules. Modules work as filters and policy enforcers that are inserted in the main event stream of the router in a pipe and filter architecture style. A special process called the graph manager controls the dynamic change (insertion and removal) of these components in the package flow of the router.
Even though the problem of routing Internet packages does not provide the full content-based filtering of a publish/subscribe model, it provide good insights on how to provide dynamic change using a modular architecture, as well as the service priority arrangement provided by the workflow model.

\subsection{Configurable distributed systems}

Software architectures and event-based systems can be combined to provide a framework to support runtime configuration. Oreizy and Taylor [20], for example, propose the use of the C2 architectural style to support these changes. Likewise, event processing languages (such as GEM) and dynamic architecture languages (as Darwin) can be used to implement configurable distributed systems in the application level [21].

\subsection{Configurable middleware}

Configurable middleware services have been described in the literature. For example, TAO [22] allows the static configuration of services or the runtime change of strategic components in a CORBA ORB. TAO can be configured to cope with different realtime constraints by selecting the appropriate implementation of each component of the ORB. It also allows the definition of configurations where unnecessary components are not present, which addresses small footprint requirements of mobile devices or special real-time constraints. The motivation of this work is similar to ours: the need to cope with different requirements of specific application domains. In the case of TAO, real-time is the main application domain.

The Apache web server is another example. It uses a pluggable architecture where modules providing different services can be added. These modules can be installed in distinct phases of the request handling, processing and response sending process. This approach has some similarities to the plug-ins in our notification server. However, differently from the pluggable publish/subscribe component in our architecture, Apache uses a very simple extension model, with no parallelism and distribution flexibility: each request and response follows the same workflow. It also does not allow the addition of new services at runtime.

\section{CONCLUSIONS AND FUTURE WORK}

In this work, we describe the design of a configurable, extensible and dynamic architecture for notification services, which provides both: the specialty necessary for the implementation of domainspecific applications and the generality necessary for supporting the requirements of a broad set of applications. The architecture provides (i) customization of the entire event notification service to meet the needs of different applications; (ii) extensibility to support the addition of new features; and (iii) dynamism allowing the introduction of these features at run-time. In order to provide these advantages, the architecture uses the following main strategies: a publish/subscribe core; the extension of this core with subscription, notification and protocol plug-ins; the ability to add consumer and producer side plug-ins; and the adoption of an extensible subscription, event, protocol and configuration languages based on XML.

Future work includes the improvement of the prototype, with the implementation of client-side plug-ins; and the execution of tests using specific configurations, comparing their performance with 
available event-notification servers. Issues as timing, event ordering assurance and scalability still need to be explored in more detail in our design. The study of approaches to optimize the subscription processing, such as the use of the RITE algorithm and other approaches [23] are also part of our future plans.

\section{ACKNOWLEDGEMENTS}

The authors thank Eric Dashofy, Marcio Dias, Santoshi D. Basaveswara, and Max Slabyak for their contributions to the design of the system. We also thank CAPES (grant BEX 1312/99-5) for the financial support. Effort sponsored by the Defense Advanced Research Projects Agency (DARPA) and Air Force Research Laboratory, Air Force Materiel Command, USAF, under agreement number F30602-00-2-0599. Funding also provided by the National Science Foundation under grant numbers CCR0205724 and 9624846. The U.S. Government is authorized to reproduce and distribute reprints for governmental purposes notwithstanding any copyright annotation thereon. The views and conclusions contained herein are those of the authors and should not be interpreted as necessarily representing the official policies or endorsements, either expressed or implied, of the Defense Advanced Research Projects Agency (DARPA), the Air Force Laboratory, or the U.S. Government.

\section{REFERENCES}

[1] D. Hilbert and D. Redmiles, "An Approach to Large-scale Collection of Application Usage Data over the Internet," presented at 20th International Conference on Software Engineering (ICSE '98), Kyoto, Japan, 1998.

[2] P. Dourish and S. Bly, "Portholes: Supporting Distributed Awareness in a Collaborative Work Group," presented at ACM Conference on Human Factors in Computing Systems (CHI '92), Monterey, California, USA, 1992.

[3] Anita Sarma and A. v. d. Hoek, "Palantír: Increasing Awareness in Distributed Software Development," presented at International Workshop in Global Software Development at ICSE'2002, Orlando, Florida, 2002.

[4] G. Cugola, E. D. Nitto, and A. Fuggeta, "The Jedi EventBased Infrastructure and Its Application on the Development of the OPSS WFMS," IEEE Transactions on Software Engineering, vol. 27, pp. 827-849, 2001.

[5] OMG, "Notification Service Specification v1.0.1," Object Management Group, 2002.

[6] R. E. Gruber, B. Krishnamurthy, and E. Panagos, "The Architecture of the READY Event Notification Service," presented at In Proceedings of the 1999 ICDCS Workshop on Electronic Commerce and Web-Based Applications, Austin, TX, USA, 1999.

[7] L. Lövstrand, "Being Selectively Aware with the Khronika System," presented at European Conference on Computer Supported Cooperative Work (ECSCW '91), Amsterdam, The Netherlands, 1991.

[8] M. Kantor and D. Redmiles, "Creating an Infrastructure for Ubiquitous Awareness," presented at Eighth IFIP TC 13 Conference on Human-Computer Interaction (INTERACT 2001), Tokyo, Japan, 2001.

[9] B. Krishnamurthy and D. S. Rosenblum, "Yeast: A General Purpose Event-Action System," IEEE Transactions on Software Engineering, vol. 21, pp. 845-857, 1995.
[10] M. Mansouri-Samani and M. Sloman, "GEM: A Generalised Event Monitoring Language for Distributed Systems," presented at IFIP/IEEE International Conference on Distributed Platforms (ICODP/ICDP'97), Toronto, Canada, 1997.

[11] A. Carzaniga, D. S. Rosenblum, and A. L. Wolf, "Design and Evaluation of a Wide-Area Event Notification Service," ACM Transactions on Computer Systems, 2001.

[12] G. Fitzpatrick, T. Mansfield, D. Arnold, T. Phelps, B. Segall, and S. Kaplan, "Instrumenting and Augmenting the Workaday World with a Generic Notification Service called Elvin," presented at European Conference on Computer Supported Cooperative Work (ECSCW '99), Copenhagen, Denmark, 1999.

[13] C. R. B. de Souza, S. D. Basaveswara, and D. F. Redmiles, "Using Event Notification Servers to Support Application Awareness," presented at International Conference on Software Engineering and Applications, Cambridge, MA, 2002.

[14] D. S. Rosenblum and A. L. Wolf, "A Design Framework for Internet-Scale Event Observation and Notification," presented at 6th European Software Engineering Conference/5th ACM SIGSOFT Symposium on the Foundations of Software Engineering, Zurich, Switzerland, 1997.

[15] P. C. Bates, "Debugging heterogeneous distributed systems using event-based models of behavior," ACM Transactions on Computer Systems, vol. 13, pp. 1-31, 1995.

[16] E. Gamma, R. Helm, R. Johnson, and J. Vlissides, Design Patterns: Elements of Reusable Object-Oriented Software: Addison-Wesley Publishing Company, 1995.

[17] R. S. Silva Filho, M. Slabyak, and D. F. Redmiles, "Webbased infrastructure for awareness based on events," presented at Workshop on Network Services for Groupware ACM Conference on Computer Supported Cooperative Work (CSCW'02), New Orleans, LA, USA, 2002.

[18] E. Kohler, R. Morris, B. Chen, J. Jannotti, and M. F. Kaashoek, "The Click modular router," ACM Transactions on Computer Systems, vol. 18, pp. 263-297, 2000.

[19] M. Rio, N. Pezzi, H. D. Meer, W. Emmerich, L. Zanolin, and C. Mascolo, "Promile: A Management Architecture for Programmable Modular Routers," presented at Open Signaling and Service Conference (OpenSIG 2001), London, UK, 2001.

[20] P. Oreizy and R. N. Taylor, "On the Role of Software Architectures in Runtime System Reconfiguration," IEE Proceedings - Software Engineering, vol. 145, pp. 137-145, 1998.

[21] M. Mansouri-Samani and M. Sloman, "A configurable event service for distributed systems," presented at Proc. Configurable Distributed Systems (ICCDS'96), Annapolis, MD, USA, 1996.

[22] D. C. Schmidt and C. Cleeland, "Applying a Pattern Language to Develop Extensible ORB Middleware," in Design Patterns and Communications, L. Rising, Ed.: Cambridge University Press, 2000.

[23] R. E. Filman and D. D. Lee, "Managing Distributed Systems with Smart Subscriptions," presented at Proc. International Conference on Parallel and Distributed Processing Techniques and Applications (PDPTA'2000), Las Vegas, Nevada, USA, 2000. 